\title{
Space-Time Adaptive Principle Component Analysis for Time-Delay Estimation
}

\author{
Felix Trötschel \\ German Aerospace Center (DLR) \\ Institute of Communications \\ and Navigation \\ Oberpfaffenhofen 82234, Germany \\ Email: felix.troetschel@dlr.de
}

\author{
Felix Antreich \\ German Aerospace Center (DLR) \\ Institute of Communications \\ and Navigation \\ Oberpfaffenhofen 82234, Germany \\ Email: felix.antreich@dlr.de
}

\author{
Josef A. Nossek \\ Technical University of Munich (TUM) \\ Institute for Circuit Theory and \\ Signal Processing \\ Munich 80333, Germany \\ Email: josef.a.nossek@tum.de
}

\begin{abstract}
In this work we present a blind approach based on space-time adaptive principle component analysis using an antenna array and a compression method based on canonical components in order to mitigate multipath for Global Navigation Satellite Systems (GNSS). First, pre-processing algorithms are used to separate (decorrelate) the different sources in the compressed space-time eigenspace and then the time-delay estimation is performed with a simple interpolation scheme in the compressed time domain. The proposed algorithm is capable of separating highly correlated and even coherent signals and approaches the respective Cramer-Rao lower bound (CRLB) for time-delay estimation in the compressed time domain.
\end{abstract}

\section{INTRODUCTION}

We consider the problem of time-delay estimation of the line-of-sight (LOS) signal for Global Navigation Satellite Systems (GNSS) in a multipath environment. The quality of the ranging data provided by a GNSS receiver largely depends on the synchronization error, that is, on the accuracy of the propagation time-delay estimation of the LOS signal. In case the LOS signal is corrupted by several superimposed delayed replicas (reflective, diffractive, or refractive multipath), the estimation of the propagation time-delay and thus the position can be severely degraded. Several techniques have been proposed in the literature for solving the multipath problem in GNSS using one antenna, see e.g. [1]-[3]. When using antenna arrays high resolution parameter estimation algorithms provide high accurate results [4]-[6], but they entail rather high complexity in the parameter estimation as multi-dimensional nonlinear problems have to be solved. Furthermore, they also require accurate model order estimation [4].

In this work we present an approach for which no multidimensional nonlinear problems need to be solved and model order estimation is not required. Instead we derive a blind approach based on space-time adaptive Principle Component Analysis (PCA) using an antenna array and a compression method based on Canonical Components (CC) with a bank of signal matched correlators [6] in order to mitigate multipath and to estimate the time-delay of the LOS signal. First, adapted block-wise pre-processing algorithms like Forward-Backward Averaging (FBA) [7] and Spatial Smoothing (SPS) [8] are used to separate (decorrelate) the different sources in the compressed space-time eigenspace. Then time-delay estimation is performed with a simple interpolation scheme after tap-wise spatial filtering in the compressed time domain. Our approach follows the principles of space-time eigenrake receivers [9], but tailored to time-delay estimation for GNSS and extends the algorithm presented in [10] to an adaptive space-time method. The proposed pre-processing schemes require that the antenna array response is left centro-hermitian. In case the array response is not left centro-hermitian, signal adaptive array interpolation methods can be applied to transform the array response to a centro-hermitian array response [11].

The proposed approach is capable of separating highly correlated and even coherent signals and approaches the respective Cramer-Rao lower bound (CRLB) for time-delay estimation in the compressed time domain.

\section{Signal Model}

In this section we define the pre- and post-correlation signal model.

\section{A. Pre-correlation Signal Model}

The complex baseband signal with bandwidth $B$ that is received by an antenna array with $M$ sensor elements is

$$
\mathbf{x}(t)=\mathbf{s}(t)+\mathbf{n}(t)=\sum_{\ell=1}^{L} \mathbf{s}_{\ell}(t)+\mathbf{n}(t),
$$

where $\mathbf{s}(t) \in \mathbb{C}^{M \times 1}$ denotes the superimposed signal replicas

$$
\mathbf{s}_{\ell}(t)=\mathbf{a}\left(\phi_{\ell}, \vartheta_{\ell}\right) \gamma_{\ell} \mathrm{e}^{j 2 \pi \nu_{\ell} t} c\left(t-\tau_{\ell}\right),
$$

$\mathbf{a}\left(\phi_{\ell}, \vartheta_{\ell}\right) \in \mathbb{C}^{M \times 1}$ defines the steering vector of an antenna array with azimuth angle $\phi_{\ell}$ and elevation angle $\vartheta_{\ell}, c\left(t-\tau_{\ell}\right)$ denotes a periodically repeated pseudo random (PR) sequence $c(t)$ with time-delay $\tau_{\ell}$, chip duration $T_{c}$, and period $T=$ $N_{c} T_{c}$ with $N_{c} \in \mathbb{N}$. We assume temporally and spatially white complex Gaussian noise $\mathbf{n}(t) \in \mathbb{C}^{M \times 1}$. In the following the parameters of the LOS signal are indicated with $\ell=1$ and the parameters of the non-LOS (NLOS) signals (multipath) with $\ell=2, \ldots, L$. We define the parameter vectors

$$
\begin{aligned}
\boldsymbol{\eta} & =\left[\operatorname{Re}\{\boldsymbol{\gamma}\}^{\mathrm{T}}, \operatorname{Im}\{\boldsymbol{\gamma}\}^{\mathrm{T}}, \boldsymbol{\phi}^{\mathrm{T}}, \boldsymbol{\vartheta}^{\mathrm{T}}, \boldsymbol{\tau}^{\mathrm{T}}\right]^{\mathrm{T}}, \\
\boldsymbol{\eta}_{\ell} & =\left[\operatorname{Re}\left\{\gamma_{\ell}\right\}, \operatorname{Im}\left\{\gamma_{\ell}\right\}, \phi_{\ell}, \vartheta_{\ell}, \tau_{\ell}\right]^{\mathrm{T}},
\end{aligned}
$$


with $\gamma=\left[\gamma_{1}, \ldots, \gamma_{L}\right]^{\mathrm{T}}, \boldsymbol{\phi}=\left[\phi_{1}, \ldots, \phi_{L}\right]^{\mathrm{T}}, \boldsymbol{\vartheta}=$ $\left[\vartheta_{1}, \ldots, \vartheta_{L}\right]^{\mathrm{T}}$, and $\boldsymbol{\tau}=\left[\tau_{1}, \ldots, \tau_{L}\right]^{\mathrm{T}}$. The spatial observations are collected in $K$ periods of the PR sequence of $N$ time instances, thus $\mathbf{x}[(k-1) N+n]=\mathbf{x}\left(((k-1) N+n) T_{s}\right)$ with $n=1,2, \ldots, N, k=1,2, \ldots, K$, and the sampling frequency $\frac{1}{T_{s}} \geq 2 B$. The channel parameters are assumed constant at least during the $k$-th period of the observation interval. Collecting the samples of the $k$-th period of the observation interval we define:

$$
\begin{array}{r}
\mathbf{X}[k]=[\mathbf{x}[(k-1) N+1], \ldots, \mathbf{x}[(k-1) N+N]], \\
\mathbf{N}[k]=[\mathbf{n}[(k-1) N+1], \ldots, \mathbf{n}[(k-1) N+N]], \\
\mathbf{S}[k ; \boldsymbol{\eta}]=[\mathbf{s}[(k-1) N+1], \ldots, \mathbf{s}[(k-1) N+N]], \\
\mathbf{S}_{\ell}\left[k ; \boldsymbol{\eta}_{\ell}\right]=\left[\mathbf{s}_{\ell}[(k-1) N+1], \ldots, \mathbf{s}_{\ell}[(k-1) N+N]\right] .
\end{array}
$$

Thus, the signal can be written in matrix notation as

$$
\mathbf{X}[k]=\mathbf{S}[k ; \boldsymbol{\eta}]+\mathbf{N}[k]=\sum_{\ell=1}^{L} \mathbf{S}_{\ell}\left[k ; \boldsymbol{\eta}_{\ell}\right]+\mathbf{N}[k]=\mathbf{A}[k] \mathbf{\Gamma}[k] \mathbf{C}[k]+\mathbf{N}[k],
$$

where

$$
\mathbf{A}[k]=\left[\mathbf{a}\left(\phi_{1}, \vartheta_{1}\right), \ldots, \mathbf{a}\left(\phi_{\ell}, \vartheta_{\ell}\right), \ldots, \mathbf{a}\left(\phi_{L}, \vartheta_{L}\right)\right] \in \mathbb{C}^{M \times L}
$$

denotes the steering matrix,

$$
\Gamma[k]=\operatorname{diag}\{\gamma\} \in \mathbb{C}^{L \times L}
$$

is a diagonal matrix whose diagonal entries are $\gamma$. Furthermore,

$$
\mathbf{C}[k]=\left[\mathbf{c}\left[k ; \tau_{1}\right] \cdots \mathbf{c}\left[k ; \tau_{\ell}\right] \cdots \mathbf{c}\left[k ; \tau_{L}\right]\right]^{\mathrm{T}} \in \mathbb{R}^{L \times N}
$$

contains the sampled and shifted $c(t)$ for each impinging wavefront

$$
\begin{aligned}
\mathbf{c}\left[k ; \tau_{\ell}\right]= & {\left[c\left(((k-1) N+1) T_{s}-\tau_{\ell}\right), \ldots, c\left(((k-1) N+n) T_{s}-\tau_{\ell}\right),\right.} \\
& \left.\ldots, c\left(((k-1) N+N) T_{s}-\tau_{\ell}\right)\right]^{\mathrm{T}} .
\end{aligned}
$$

In general $\left\|\mathbf{c}\left[k ; \tau_{\ell}\right]\right\|_{2}^{2} \neq N$ for all $\tau_{\ell}$, however in many cases ${ }^{1}$ we can assume that $\left\|\mathbf{c}\left[k ; \tau_{\ell}\right]\right\|_{2}^{2} \approx N, \forall \tau_{\ell} \forall k$, if additionally $N \geq N_{c}$ and $N / N_{c} \in \mathbb{N}$ we get $\mathbf{c}\left[k ; \tau_{\ell}\right]=\mathbf{c}\left(\tau_{\ell}\right), \forall k$.

\section{B. Post-correlation Signal Model}

In this work we apply a compression based on CC [6], a bank of $Q$ signal matched correlators at the output of each antenna. Thus, the signal at the output of the $q$-th correlator at each antenna element with $q=1, \ldots, Q$ can be given as

$$
\mathbf{y}_{q}[k]=\mathbf{X}[k] \frac{1}{N} \mathbf{c}^{*}\left[k ; \kappa_{q}\right] \in \mathbb{C}^{M \times 1},
$$

where $\kappa_{q}$ denotes the time-delay for the correlator tap $q$. We define the post-correlation data matrix which comprises all outputs of each bank of correlators for each antenna element as

$$
\mathbf{Y}=\left[\begin{array}{ccc}
\mathbf{y}_{1}[1] & \cdots & \mathbf{y}_{1}[K] \\
& \vdots & \\
\mathbf{y}_{Q}[1] & \cdots & \mathbf{y}_{Q}[K]
\end{array}\right] \in \mathbb{C}^{M Q \times K} .
$$

\footnotetext{
${ }^{1}$ e.g. in case of GPS C/A PR sequences with bandwidth $B \geq 1.023 \mathrm{MHz}$.
}

Furthermore, we define

$$
\begin{aligned}
\overline{\mathbf{s}}[k] & =\frac{1}{N} \operatorname{vec}(\mathbf{A}[k] \mathbf{\Gamma}[k] \mathbf{C}[k] \mathbf{Q}[k]) \in \mathbb{C}^{M Q \times 1}, \\
\overline{\mathbf{n}}[k] & =\frac{1}{N} \operatorname{vec}(\mathbf{N}[k] \mathbf{Q}[k]) \in \mathbb{C}^{M Q \times 1}, \\
\overline{\mathbf{s}}(\boldsymbol{\eta}) & =[\overline{\mathbf{s}}[1], \ldots, \overline{\mathbf{s}}[k], \ldots, \overline{\mathbf{s}}[K]] \in \mathbb{C}^{M Q \times K}, \\
\overline{\mathbf{N}} & =[\overline{\mathbf{n}}[1], \ldots, \overline{\mathbf{n}}[k], \ldots, \overline{\mathbf{n}}[K]] \in \mathbb{C}^{M Q \times K},
\end{aligned}
$$

where

$$
\mathbf{Q}[k]=\left[\mathbf{c}\left[k ; \kappa_{1}\right], \ldots, \mathbf{c}\left[k ; \kappa_{q}\right], \ldots, \mathbf{c}\left[k ; \kappa_{Q}\right]\right] \in \mathbb{R}^{N \times Q}
$$

denotes the reference sequence matrix of the bank of correlators and $\operatorname{vec}(\cdot)$ describes the vec-operator. Finally we can write

$$
\mathbf{Y}=\overline{\mathbf{S}}(\boldsymbol{\eta})+\overline{\mathbf{N}}
$$

and consequently the post-correlation space-time covariance matrix can be given as

$$
\mathbf{R}_{\mathbf{y y}}=\mathrm{E}\left[\mathbf{y}[k] \mathbf{y}^{\mathrm{H}}[k]\right],
$$

where $\mathbf{y}[k]=\left[\mathbf{y}_{1}[k]^{\mathrm{T}}, \mathbf{y}_{2}[k]^{\mathrm{T}}, \ldots, \mathbf{y}_{q}[k]^{\mathrm{T}}, \ldots, \mathbf{y}_{Q}[k]^{\mathrm{T}}\right]^{\mathrm{T}}$ describes all correlator outputs for the $k$-th period. With $\mathrm{E}\left[\overline{\mathbf{s}}[k] \overline{\mathbf{n}}^{\mathrm{H}}[k]\right]=\mathbf{0}$ we can write

$$
\mathbf{R}_{\mathbf{y y}}=\mathbf{R}_{\overline{\mathbf{s}} \overline{\mathbf{s}}}+\mathbf{R}_{\overline{\mathbf{n}} \overline{\mathbf{n}}}
$$

with

$$
\begin{aligned}
\mathbf{R}_{\overline{\mathbf{s}} \overline{\mathbf{s}}} & =\mathrm{E}\left[\overline{\mathbf{s}}[k] \overline{\mathbf{s}}^{\mathrm{H}}[k]\right] \\
& =\mathrm{E}\left[\frac{1}{N^{2}} \operatorname{vec}(\mathbf{A}[k] \boldsymbol{\Gamma}[k] \mathbf{C}[k] \mathbf{Q}[k]) \operatorname{vec}^{\mathrm{H}}(\mathbf{A}[k] \boldsymbol{\Gamma}[k] \mathbf{C}[k] \mathbf{Q}[k])\right] \\
& =\left(\mathbf{I}_{Q} \otimes \mathbf{A}\right) \frac{1}{N^{2}} \operatorname{vec}(\boldsymbol{\Gamma} \mathbf{C} \mathbf{Q}) \operatorname{vec}^{\mathrm{H}}(\boldsymbol{\Gamma} \mathbf{C} \mathbf{Q})\left(\mathbf{I}_{Q} \otimes \mathbf{A}\right)^{\mathrm{H}} \\
& =\left(\mathbf{I}_{Q} \otimes \mathbf{A}\right) \mathbf{R}_{s}\left(\mathbf{I}_{Q} \otimes \mathbf{A}\right)^{\mathrm{H}},
\end{aligned}
$$

in case the channel parameters and the time-delays of the bank of correlators $\kappa_{q}$ are constant within the observation interval of $K$ periods. Here, $\mathbf{I}_{Q}$ denotes an $Q \times Q$ identity matrix. The noise covariance matrix can be given as

$$
\mathbf{R}_{\overline{\mathbf{n}} \overline{\mathbf{n}}}=\mathrm{E}\left[\overline{\mathbf{n}}[k] \overline{\mathbf{n}}^{\mathrm{H}}[k]\right]=\frac{\sigma_{n}^{2}}{N^{2}}\left(\mathbf{G} \otimes \mathbf{I}_{M}\right),
$$

where $\otimes$ denotes the Kronecker product and $\mathbf{G}=\mathbf{Q}^{\mathrm{T}} \mathbf{Q}^{*}$.

After pre-whitening with $\left(\mathbf{G} \otimes \mathbf{I}_{M}\right)^{-\frac{1}{2}}$ we get

$$
\begin{aligned}
\tilde{\mathbf{R}}_{\mathbf{y y}} & =\left(\mathbf{G} \otimes \mathbf{I}_{M}\right)^{-\frac{1}{2}} \mathbf{R}_{\overline{\mathbf{s}} \mathbf{s}}\left(\mathbf{G} \otimes \mathbf{I}_{M}\right)^{-\frac{\mathrm{H}}{2}}+\frac{\sigma_{n}^{2}}{N^{2}} \mathbf{I}_{M Q} \\
& =\left(\mathbf{G}^{-\frac{1}{2}} \otimes \mathbf{A}\right) \mathbf{R}_{s}\left(\mathbf{G}^{-\frac{1}{2}} \otimes \mathbf{A}\right)^{\mathrm{H}}+\frac{\sigma_{n}^{2}}{N^{2}} \mathbf{I}_{M Q}
\end{aligned}
$$

\section{Space-Time Adaptive Principle Component ANALYSIS AND TIME-DELAY ESTIMATION}

When two or more signals (LOS signal plus several NLOS signals) which are highly correlated or even coherent are received by the antenna array, the corresponding components in an orthogonal basis (e.g. eigenvectors) of $\tilde{\mathbf{R}}_{\mathbf{y y}}$ cannot be separated. Thus, a PCA using a corresponding orthogonal basis (e.g. eigenspace) would fail. Techniques such as FBA [7] and SPS [8] can be applied to decorrelate the signals and hence to smoothen the corresponding orthogonal basis decomposition (e.g. eigenspace) and to enable a PCA with subsequent precise time-delay estimation of the LOS signal. In order to apply FBA 
and SPS the array steering matrix must be left centro-hermitian such that

$$
\mathbf{A}=\boldsymbol{\Pi}_{M} \mathbf{A}^{*} \text { with } \boldsymbol{\Pi}_{M}=\left[\begin{array}{lll} 
& & 1 \\
& & \\
1 & &
\end{array}\right] \in \mathbb{R}^{M \times M} .
$$

With (27) one can observe from (26) that $\tilde{\mathbf{R}}_{\mathbf{y y}}$ is block-wise centro-hermitian such that

$$
\tilde{\mathbf{R}}_{\mathbf{y y}}=\boldsymbol{\Pi}_{M}^{\otimes} \tilde{\mathbf{R}}_{\mathbf{y y}}^{*} \Pi_{M}^{\otimes}
$$

with

$$
\mathbf{\Pi}_{M}^{\otimes}=\mathbf{I}_{Q} \otimes \boldsymbol{\Pi}_{M} \in \quad \mathbb{N}_{0}^{M Q \times M Q} .
$$

In the following subsections we describe block-wise spacetime FBA and SPS for an estimate of the pre-whitened spacetime covariance matrix $\hat{\tilde{\mathbf{R}}}_{\mathbf{y y}}$ in order to decorrelate highly correlated or even coherent signals and to finally perform timedelay estimation of the LOS signal. An estimate of the prewhitened space-time covariance matrix can be given as

$$
\hat{\tilde{\mathbf{R}}}_{\mathbf{y y}}=\left(\mathbf{G} \otimes \mathbf{I}_{M}\right)^{-\frac{1}{2}} \frac{1}{K} \mathbf{Y} \mathbf{Y}^{\mathrm{H}}\left(\mathbf{G} \otimes \mathbf{I}_{M}\right)^{-\frac{\mathrm{H}}{2}}
$$

\section{A. Block-wise Forward-Backward Averaging (FBA)}

As the pre-whitened space-time covariance matrix has a block-wise centro-hermitian structure we can apply the following block-wise FBA

$$
\hat{\tilde{\mathbf{R}}}_{\mathbf{y y}}^{\mathrm{FBA}}=\frac{1}{2}\left(\hat{\tilde{\mathbf{R}}}_{\mathbf{y y}}+\boldsymbol{\Pi}_{M}^{\otimes} \hat{\tilde{\mathbf{R}}}_{\mathbf{y y}}^{*} \mathbf{\Pi}_{M}^{\otimes}\right),
$$

because of the Kronecker structure of the extended exchange matrix, the FBA does not depend on the time synchronization between the signal and the bank of correlators. This bockwise FBA doubles the number of available observations and enables to separate of two coherent or highly correlated signals through a decorrelation without decreasing the effective size of the antenna array.

\section{B. Block-wise Spatial Smoothing (SPS)}

In this work we derive a block-wise 2-D SPS scheme for the space-time covariance matrix considering an Uniform Rectangular Arrays (URAs) with $M_{x} \times M_{y}$ elements. We define linear subarrays in $\mathrm{x}$ - and $\mathrm{y}$-direction with the same number of sensors. Therefore, we get the number of sensors for one subarray in x-direction $M_{s u b_{x}}=M_{x}-L_{x}+1$, where $L_{x}$ defines the number of linear subarrays in x-direction. In our case $M_{s u b_{x}}$ is equal to the number of sensors in y-direction $M_{s u b_{y}}$ and also $L_{x}$ is equal to $L_{y}$. Then the number of rectangular subarrays is $L_{s}=L_{x} L_{y}$ and each subarray array is containing $M_{s u b}=M_{s u b_{x}} M_{s u b_{y}}$ sensor elements.

Since in our case all subarrays have the same size, only the calculations for one direction are needed for the selection matrices:

$$
\mathbf{J}_{\ell_{x}}=\left[\begin{array}{lll}
\mathbf{0} & \mathbf{I}_{M_{\text {subx }}} & \mathbf{0}
\end{array}\right] \forall 1 \leq \ell_{x} \leq L_{x} .
$$

In case of uniform subarrays $\mathbf{J}_{\ell_{y}}$ is equal to $\mathbf{J}_{\ell_{x}}$. Then the block-wise 2-D SPS is obtained by a combination of the selection matrices of two different directions. Thus, we get

$$
\mathbf{J}_{\ell_{x}, \ell_{y}}^{\text {Bank }}=\mathbf{I}_{Q} \otimes\left(\mathbf{J}_{\ell_{y}} \otimes \mathbf{J}_{\ell_{x}}\right) \in \mathbb{N}_{0}^{Q M_{s u b} \times Q M} .
$$

Then, the spatially smoothed pre-whitened space-time covariance matrix is

$$
\hat{\tilde{\mathbf{R}}}_{\mathbf{y y}}^{\mathrm{SPS}}=\frac{1}{L_{s}} \sum_{\ell_{y}=1}^{L_{y}} \sum_{\ell_{x}=1}^{L_{x}} \mathbf{J}_{\ell_{x}, \ell_{y}}^{\mathrm{Bank}} \hat{\tilde{\mathbf{R}}}_{\mathbf{y y}}\left(\mathbf{J}_{\ell_{x}, \ell_{y}}^{\mathrm{Bank}}\right)^{\mathrm{T}} \in \mathbb{C}^{Q M_{s u b} \times Q M_{s u b}} .
$$

Both, block-wise FBA and SPS can be used alone or in combination to improve the results for the next processing steps.

\section{Time-Delay Estimation}

After separating the different signal components in the eigenspace using block-wise FBA and SPS we can use the eigenvector $\mathbf{w} \in \mathbb{C}^{Q M \times 1}$ related to the largest eigenvalue of $\hat{\mathbf{R}}_{\mathbf{y y}}^{\mathrm{FBA}+\mathrm{SPS}}$ to estimate the time-delay $\tau_{1}$ by a spatial filtering of each tap in the compressed time domain and subsequent interpolation. This tap-wise filtering can be given as

$$
\mathbf{p}[k]=\mathbf{W}^{\mathrm{H}} \mathbf{J}_{1,1}^{\text {Bank }}(\mathbf{G} \otimes \mathbf{I})^{-\frac{1}{2}} \mathbf{y}[k] \quad \in \mathbb{C}^{Q \times 1},
$$

where

$$
\mathbf{W}=\left[\left(\mathbf{I}_{Q} \otimes \mathbf{I}_{M_{s u b}}\right) \square \mathbf{w}^{\mathrm{T}}\right]^{\mathrm{T}} \quad \in \mathbb{C}^{Q M_{s u b} \times Q} .
$$

Here $\square$ denotes the Khatri-Rao product. Based on $\mathbf{p}[k]$ and a cubic spline interpolation we can derive the cost function $P(\tau)$ and then estimate the time-delay of the LOS signal by

$$
\begin{gathered}
\hat{\tau}_{1}=\arg \max _{\tau}\{P(\tau)\} . \\
\text { IV. SIMULATIONS }
\end{gathered}
$$

In this section we assess the performance of our proposed approach by computer simulations. For each set of signal parameters, we perform 4000 Monte Carlo runs and calculate the root mean square estimation error (RMSE) and the CRLB.

First we consider a scenario with $L=2$. We assume a GPS C/A signal with $B=1.023 \mathrm{MHz}$ and a centro-hermitian URA with $M=3 \times 3$ isotropic sensor elements and $\lambda / 2$ spacing. The DOAs of the two paths are: $\vartheta_{1}=75^{\circ}, \phi_{1}=25^{\circ}$, $\vartheta_{2}=25^{\circ}$, and $\phi_{2}=144^{\circ}$. The Carrier-to-Noise density ratio $\left(C / N_{0}\right)$ for the LOS signal is $42 \mathrm{~dB}-\mathrm{Hz}$ and for the multipath signal is $40 \mathrm{~dB}$. We assume that the observation interval is $K=10$ periods and that the channel parameters are assumed constant within the observation interval. We define a relative time-delay between the two signals as $\Delta \tau=\left|\tau_{1}-\tau_{2}\right| / T_{c}$. For the bank signal matched of correlators we assume $Q=11$ and that its taps are distributed equidistantly within the interval $\left[-T_{c} ; T_{c}\right]$. The synchronization error between the centered correlator of the bank of correlators and $\tau_{1}$ is denoted as $\Delta \tau_{\text {Bank }}=\left|\tau_{1}-\kappa_{6}\right|$ (for $Q=11$ ) and we assume that it is uniformly distributed within the interval $\left[-T_{c} ; T_{c}\right]$. Thus, in our simulations as well as in the respective RMSE 
and the expectation of the square root of the derived CRLB $\mathrm{E}\{\sqrt{\mathrm{CRLB}}\}$, the compression loss introduced by the bank of correlators with respect to its synchronization error $\Delta \tau_{\text {Bank }}$ is considered. In Fig. 1 and Fig.2 the RMSE for $\tau_{1}$ consid-

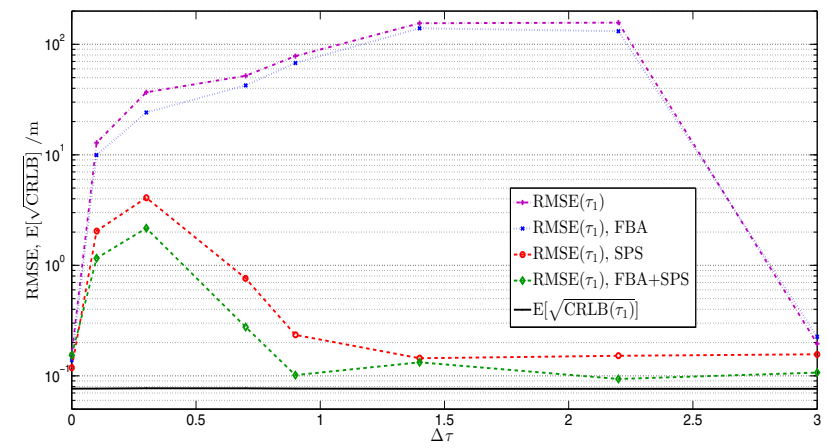

Fig. 1. RMSE and $\mathrm{E}|\sqrt{\mathrm{CRLB}}|$ versus $\Delta \tau$ with $\left|\arg \left(\gamma_{1}\right)-\arg \left(\gamma_{2}\right)\right|=\pi / 3$

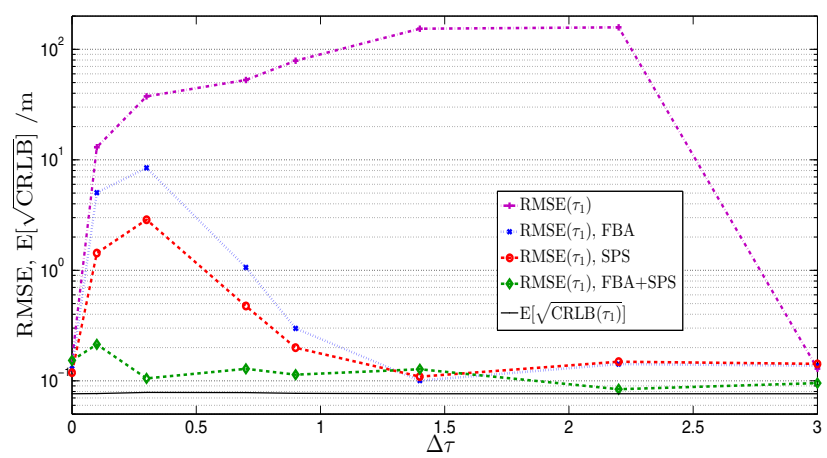

Fig. 2. RMSE and $\mathrm{E}|\sqrt{\mathrm{CRLB}}|$ versus $\Delta \tau$ with $\left|\arg \left(\gamma_{1}\right)-\arg \left(\gamma_{2}\right)\right|=\pi / 2$

ering no pre-processing, FBA, SPS, and FBA+SPS as well as $\mathrm{E}\{\sqrt{\mathrm{CRLB}}\}$ versus $\Delta \tau$ are depicted for the cases with $\left|\arg \left(\gamma_{1}\right)-\arg \left(\gamma_{2}\right)\right|=\pi / 3$ and $\left|\arg \left(\gamma_{1}\right)-\arg \left(\gamma_{2}\right)\right|=\pi / 2$. For the SPS we choose $L_{s}=4$ with URAs as subarrays with $M_{s u b_{x}}=2$ and $M_{s u b_{y}}=2$. We can observe that the performance of FBA improves for an increasing relative phase between the two paths while SPS in general is dependent on the number of subarrays $L_{s}$ and the spatial separation of the paths.

In Fig.3 simulations with various numbers of multipath signals are depicted. The $C / N_{0}$ for the LOS signal is $42 \mathrm{~dB}-\mathrm{Hz}$. We assume for the multipath signals that $\left|\gamma_{\ell}\right|^{2}=\left|\gamma_{2}\right|^{2} e^{-\alpha(\ell-2)} \forall \ell \geq 2$, with $\alpha=0.2051$ and $10 \log _{10}\left(\frac{\left|\gamma_{1}\right|^{2}}{\left|\gamma_{2}\right|^{2}}\right)=-4 \mathrm{~dB}$. The relative phase $\mid \arg \left(\gamma_{1}\right)-$ $\arg \left(\gamma_{\ell}\right) \mid$ varies between $[0.4 \pi, \pi / 2]$ and the relative time-delay $\Delta \tau$ starts from 0.3 to 2.65 in 0.1237 steps for increasing $\ell$. Here, we have used a $4 \times 4$ URA with $L_{s}=9$ and $M_{s u b_{x}}=M_{s u b_{y}}=2$.

\section{CONClusion}

In this work we have derived a blind approach based on space-time adaptive PCA using a compression method

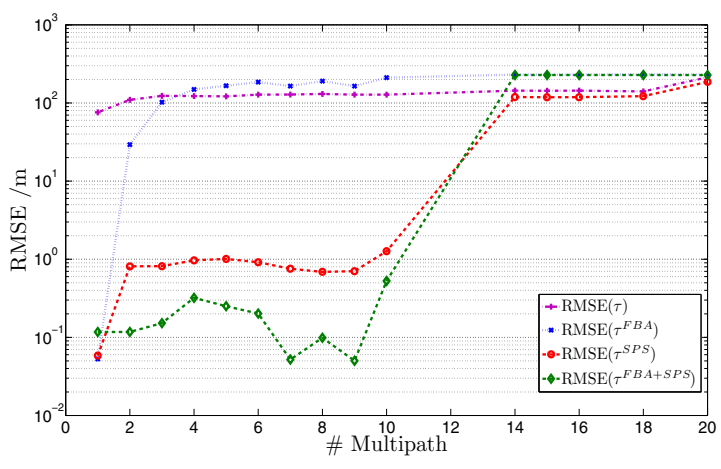

Fig. 3. RMSE versus number of multipath signals

based on CC with a bank of signal matched correlators in order to mitigate multipath and to estimate the time-delay of the LOS signal. The proposed approach provides good performance even when several coherent sources are present while demanding reasonably low computational complexity.

\section{REFERENCES}

[1] R. D. J. van Nee and P. C. Fenton, "The Multipath Estimating Delay Lock Loop Approaching Theoretical Accuracy Limits ", in Proceedings of IEEE Position Location and Navigation Symposium, Las Vegas, NV, USA, April 1994.

[2] P. Closas, C. Fernandes-Prades, and J. A. Fernandes-Rubio, "A Particle Filtering Tracking Algorithm for GNSS Synchronization Using Laplaces Method," in Proceedings of the 33rd IEEE International Conference on Acoustics, Speech and Signal Processing ICASSP, Las Vegas, U.S.A., April 2008.

[3] B. Krach, M. Lentmaier, and P. Robertson, "Joint Baysian Positioning and multipath Mitigation in GNSS," in Proceedings of the 33rd IEEE International Conference on Acoustics, Speech and Signal Processing ICASSP, Las Vegas, U.S.A., April 2008.

[4] F. Antreich, J. A. Nossek, G. Seco-Granados, and L. A. Swindlehurst, "The Extended Invariance Principle for Signal Parameter Estimation in an Unknown Spatial Field," IEEE Transactions on Signal Processing, vol. 59, no. 7, July 2011.

[5] G. Seco-Granados, J. A. Fernández-Rubio, and C. Fernández-Prades, "ML Estimator and Hybrid Beamformer for Multipath and Interference Mitigation in GNSS Receivers," IEEE Transactions on Signal Processing, vol. 53, no. 3, March 2005.

[6] J. Selva-Vera, Efficient Multipath Mitigation in Navigation Systems, Ph.D. thesis, Department of Signal Signal Theory and Communications, Universitat Politècnica de Catalunya, Spain, 2004.

[7] S.U. Pillai and B. H. Kwon, "Forward/backward Spatial Smoothing Techniques for Coherent Signal Identification ," IEEE Transactions on Acoustics, Speech and Signal Processing, vol. 37, pp. 8-9, January 1989.

[8] T. J. Shan, M. Wax, and T Kailath, "Spatial Smoothing Approach for Location Estimation of Coherent Sources ," IEEE Transactions on Acoustics, Speech and Signal Processing, vol. 33, pp. 806-811, August 1985.

[9] C. Brunner, M. Haardt, and J. A. Nossek, "On space-time rake receiver structures for WCDMA," in Proceedings Proc. 33rd Asilomar Conf. on Signals, Systems, and Computers, Pacific Grove, CA, U.S.A., October 1999.

[10] M. Sgammini, F. Antreich, L. Kurz, M. Meurer, and T.G. Noll, "Blind Adaptive Beamformer Based on Orthogonal Projections for GNSS," in Proceedings of ION GNSS 2012, Nashville, TN, U.S.A., September 2012.

[11] M. A. M. Marinho, F. Antreich, J. P. C. L. da Costa, and J. A. Nossek, "A Signal Adaptive Array Interpolation Approach with Reduced Transformation Bias for DOA estimation of Highly Correlated Signals," in Proceedings of the IEEE International Conference on Acoustics, Speech, and Signal Processing (ICASSP) 2014, Florence, Italy, May 2014. 